\title{
Erratum to: Why does Sleep Stop Migraine?
}

\author{
Marcelo E. Bigal • Richard J. Hargreaves
}

Published online: 7 January 2014

(C) Springer Science+Business Media New York 2014

Erratum to: Current Pain and Headache Reports, September 2013, 17:369

DOI: 10.1007/s11916-013-0369-0

The section titled Subcortical Structures Relevant to Migraine involved in Sleep should read as follows:

Our comprehension of the physiology of the daily cycles of sleep and wakefulness, as well as the neuronal circuitry and molecular basis of the biological clock that underpins human circadian rhythms (daily sleep-wake cycles), has increased substantially in recent years.

The wake promotion systems and the sleep promotion system are temporally controlled by the endogenous circadian pacemaker contained within the suprachiasmatic nuclei (SCN) that functions autonomously as the brain's master clock. The SCN receives information about light and dark periods from melanopsin expressing retinal ganglion cells during the day (the light entrainment system) and melatonin from the pineal gland at night to reset the human 24.5 hour circadian rhythm to our 24 hour day length. The molecular basis of the clock function involves transcriptional and translational regulation of the expression of genes such as period, clock, Bmal and cryptochrome that when mutated or deleted disrupt circadian rhythm cycles [49].

Daily oscillations in SCN activity signal through the subparaventricular zone and the dorsal medial hypothalamus to both GABA-ergic neurons of the ventrolateral pre-optic

The online version of the original article can be found at doi:http:// dx.doi.org/10.1007/s11916-013-0369-0.

M. E. Bigal $(\bowtie)$

Labrys Biologics, 1810 Gateway Drive, Suite 230, San Mateo, CA 94404, USA

e-mail: mbigal@labrysbiologics.com

R. J. Hargreaves

Preclinical Research, Merck Inc., Whitehouse Station, NJ, USA area of the hypothalamus (VLPO) and the wake promoting orexinergic neurons of the lateral hypothalamus. GABA-ergic neurons promote sleep by sending inhibitory projections both to orexin neurons as well as brain stem nuclei such as locus ceruleus (norepinephrine), Raphe nucleus (serotonin) and tuberomamillary nucleus (histamine) that together with cholinergic projections from the basal forebrain form the ascending arousal systems. Attenuation of this inhibitory signal and increased excitatory input from the SCN to orexin secreting neurons that innervate these monoaminergic brain stem arousal nuclei promotes waking and somatosensory function. [50]. Increasing evidence suggests that human circadian rhythms are regulated by a thalamic switch that can be activated or deactivated to regulate sensory information reaching the cerebral cortex and the degree of consciousness [51]. The transition from sleep to wakefulness comes as thalamocortical pathways activate the cortex that has been primed by neurotransmitters (serotonin, histamine, norepinephrine) released from ascending pathways originating in the brain stem. The extent of information passing to the cortex through the thalamus is "gated" based on the arousal level of the central nervous system that is in turn set by outputs from the reticulo-activating systems (RAS) of the brain stem and hypothalamus.

Migraine is often triggered by stimuli that activate the RAS such as environmental stimuli, exercise, changes in levels of stress, fatigue, sleep deprivation or poor sleep hygiene [52, 53] suggesting that the propensity to sleep during migraine could be an endogenous homeostatic response that can reduce the intensity or frequency of migraine headaches. The mechanisms through which physiological sleep achieves an antimigraine effect are likely to be diverse. It is well known that sleep influences output from the cardiovascular, sensory, 
motor, endocrine and thermoregulatory systems $[54,55]$ and that baseline autonomic physiology changes during sleep as a function of time and sleep stage [56]. With progressively deepening sleep stages there is a relative shift from sympathetic to parasympathetic tone with a decrease in blood pressure and reduction in muscle tone that is regained upon waking. Paradoxically, during REM sleep there is atonia. Interestingly lifestyle, mind/body and behavioral treatments for migraine also manipulate many of these autonomic physiologies and have evidence of effectiveness for the treatment of migraine in some patients yet common sedative-hypnotic medications used to treat primary insomnia have little efficacy [57].

\section{The section titled Novel Neuropeptide Arousal Systems in the Brain should read as follows:}

Recent advances in neuroscience research have focused on a small number of neurons that are clustered in the lateral hypothalamus [58] and understanding their pharmacology and physiology in health and disease. In 1998 it was discovered that these neurons, now described as orexinergic, produced a pair of closely related neuropeptides that were named orexin A (OX$\mathrm{A})$ and $\mathrm{B}(\mathrm{OX}-\mathrm{B})$ by one group of investigators and hypocretin 1 and 2 by the other. The OX-A and OX-B neuropeptides are generated from the pre-pro-orexin peptide precursor encoded by the HCRT gene, packaged into dense core vesicles of lateral hypothalamic neurons and are released via exocytosis from orexinergic neurons when they are activated. The orexin peptides bind to two different orexin receptors OX-1R and OX2R. The binding affinity of OX-A and OX-B for OX2R is similar, but the affinity of OX-B for OX1R is approximately 10 times lower than for OX2R. The primary mechanism of signaling from both OX-1R and OX-2R is through G-protein activation resulting in an elevation of intracellular $\mathrm{Ca} 2+$ from intracellular stores and neuronal activation [59].

The orexin neurons of the hypothalamus fire during waking and are quiescent during sleep [60]. They innervate the brain regions that integrate sensory and autonomic systems with sleep behaviors [61]. OX-1 R and OX-2R are widely expressed throughout the cerebral cortex. OX2R are selectively expressed in the tuberomamillary nuclei (TMN) of the hypothalamus, and are thought to be predominantly involved in promoting arousal by triggering the release of histamine in cortical and sub-cortical brain areas. OX1R and OX2R are coexpressed in brain stem nuclei responsible for sleep/wake control and sub-cortical arousal, the exception being the noradrenergic locus ceruleus where OX1R are selectively expressed and are involved in REM sleep gating [62]. Interestingly (see above) it is these brain-stem regions that have been shown to be activated during migraine attacks.

Deficiencies in the expression of orexin peptide or its receptors lead to dysfunction in the mechanisms that determine sleep and arousal states in genetically altered mice, dogs and humans. Studies on human sleep syndromes where there is a loss of ability to control sleep states (narcolepsy) have shown the central involvement of impairments in the orexin producing systems [63].

In addition to their importance in wakefulness, recent studies in animals have linked the orexins to modulation of nociceptive processing but the pharmacology of these interactions is still not fully understood and can be conflicting [64]. This may be because many of the studies have used direct injection of OX-A and OX-B at supra-pharmacologic doses so selectivity for OX-1R and OX2R is lost confounding allocation of receptor specificity to their pro and anti-nociceptive effects. Moreover, it is well known that OX-B is relatively unstable in vivo compared to OX-A especially after i.v. administration such that its effects or lack of them may be missed or underestimated after exogenous administration. Finally the selectivity of some of the early small molecule orexin antagonists may not be sufficient to provide confirmation of mechanism of action and new more selective pharmacologic tools will probably be required to provide definitive data [64]. This having been said in non-clinical studies, OX-1R has been localized to the spinal cord and dorsal root ganglion [65].OX-A and OX-B have been shown to be analgesic when given i.c.v. and intrathecally but not subcutaneously in line with their peptidic nature [66]. In animals, the efficacy of orexin-A acting at OX-1R (as defined by blockade of the response with the small molecule OX-1R antagonist SB334867, was found to be similar to that of morphine [67].

The orexins have also been recently linked with a possible role in migraine. Preclinical evidence suggests that OX-A acting at OX-1 receptors can modulate trigeminovascular nociceptive physiology such as dural vasodilatation [68] and input to the trigeminal nucleus caudalis after electrical stimulation [69]. Stimulation of the superior saggital sinus, an experimental maneuver for activating pathways relevant to migraine was shown to activate neurons in the posterior hypothalamus [70] Injection of OX-A or OX-B into the posterior hypothalamus has been shown to differentially modulate nociceptive dural input to the TNC. Micro-injection of OX-A elicited an anti-nociceptive effect reducing A and C fiber responses to dural stimulation and spontaneous activity, in agreement with other analgesia studies whereas interestingly OX-B activation elicited the opposite "pro-nociceptive" effect enhancing nociceptive transmission and convergent sensory responses to facial thermal stimulation [71]. Most recently novel dual OX-1R/OX-2R receptor antagonists that have been developed for the treatment of primary insomnia [72] have been used to probe the pharmacology of the trigeminal system rather than the peptide orexin agonists. The dual orexin antagonists were shown [73] to inhibit electrically evoked trigeminal nociceptive transmission and neuronal activity in the trigeminocervical complex supporting a pro- 
nociceptive role for orexins in trigeminal pain pathways. Delineation of the exact orexin receptor responsible for this effect awaits the study of OX-1R and OX-2R selective antagonists.

Given these data suggesting that orexins could promote trigeminovascular nociception, the propensity to sleep during migraine, and the possibility that migraine could be triggered by stimuli that activate the hypothalamus and increase orexin activity it has been hypothesized that orexin antagonists may have efficacy in the treatment of migraine. There are however several important points to consider that could impact the design and outcome of clinical trials of this hypothesis. First, the use of current dual orexin receptor antagonists will be limited by their marked sleep promoting effects such that they cannot be given to treat migraines during the day unless induction of sleep is clinically acceptable or anti-migraine effects are anticipated at much lower doses than used for treatment of primary insomnia. As a consequence, they will be dosed at bed time, to avoid day-time drug exposure and impairment of functioning, making initial studies a test (in contrast to the acute non-clinical studies conducted to date) of migraine prevention rather than a test of orexin antagonism as a novel migraine treatment. Second, the development of orexin receptor selective antagonists that lack sleep promoting properties may be required for clinical trials and this needs novel molecules to be developed to explore the relative contribution of OX-1R and OX-2R to signaling in trigeminal nociceptive processing compared to sleep/wake pathways. Third, in order to avoid diluting clinically meaningful signals, it may be necessary to focus trials on migraine sufferers with co-morbid chronic sleep disorders and perhaps those with aura as evidence of pronounced CSD to complete clinical proof of concept testing.

\section{The following references are cited in the updated sections shown above:}

\section{References}

49. Hastings MH, Herzog ED. Clock genes, oscillators and cellular networks in the suprachiasmatic nuclei. J Biol Rhythms 2004; 400-413.

50. Espana RA, Scammell TE. Sleep neurobiology from a clinical perspective. Sleep. 2011;34:845-58.

51. Saper CB, Scammell TE, Lu J. Hypothalamic regulation of sleep and circadian rhythms. Nature. 2005;437:1257-63.

52. Buijs RM, van Eden CG. The integration of stress by the hypothalamus, amygdala and prefrontal cortex: balance between the autonomic nervous system and the neuroendocrine system. Prog Brain Res. 2000; $126: 117-32$

53. Overeem S, van Vliet JA, Lammers GJ, Zitman FG, Swaab DF, Ferrari MD. The hypothalamus in episodic brain disorders. Lancet Neurol. 2002;1:437-44.
54. Chouchou F, Pichot V, Perchet C, Legrain V, Garcia-Larrea L, Roche $\mathrm{F}$, et al. Autonomic pain responses during sleep. A study of heart rate variability. Eur J Pain. 2011;15:554-60.

55. Schwimmer H, Stauss HM, Abboud F, Hishino S, Mignot E, Zeitzer JM. Effects of sleep on the cardiovascular and thermoregulatory systems: a possible role for the hypocretins. J Appl Physiol. 2010;109:1053-63.

56. Trinder J, Kleiman J, Carrington M, Smith S, Breen S, Tan N, et al. Autonomic activty during human sleep as a function of time and sleep stage. J Sleep Res. 2001;10:253-64.

57. Wells RE, Loder E. Mind/body and behavioral treatments. The evidence and approach. Headache. 2012;52:70-5.

58. Siegel JM. Hypocretin (orexin): role in normal behavior and neuropathology. Annu Rev Psychol. 2004;55:125-48.

59. Gotter A, Webber AL, Coleman PJ, Renger JJ Winrow CJ. International union of basic and clinical pharmacology. LXXXVI. Orexin receptor function, nomencalture and pharmacology. Pharmacol Rev. 2012;64:389-420.

60. Lee MG, Hassani OK, Jones BE. Discharge of identified orexin/ hypocretin neurons across the sleep-waking cycle. J Neurosci. 2005;25:6716-20.

61. De Lecea L. Hypocretins and the neurobiology of sleep-wake mechanisms. Progr Brain Res. 2012;198:15-23.

62. Sakurai T. The neuronal circuit of orexin (hypocretin): maintaining sleep and wakefulness. Nat Rev Neurosci. 2007;8:171-81.

63. Hungs M, Mignot E. Hypocretin/orexin, sleep and narcolepsy. BioEssays. 2001;23:397-408.

64. Al G, Roecker AJ, Hargreaves R, Coleman PJ, Winrow CJ, Renger JJ. Orexin receptors as therapeutic targets. Prog Brain Res. 2012;198: $163-88$.

65. Kajiyama S, Kawamoto M, Shiraishi S, et al. Spinal orexin-1 receptors mediate anti-hyperalgesic effects of intrathecally-administered orexins in diabetic neuropathic pain model rats. Brain Res. 2005 May 17;1044:76-86

66. Mobarakeh JI, Takahashi K, Sakurada S, Nishino S, Watanabe H, Kato M, et al. Enhanced nociception by intraceebroventricularly and intrathecally adminsitered orexin A and B ( hypocretin 1 and 2) in mice. Peptides. 2005;26:767-77.

67. Bingham S, Davey PT, Babbs AJ, Irving EA, Sammons MJ, Wyles $\mathrm{M}$, et al. Orexin-A an hypothalamic peptide with anlgesic properties. Pain. 2001;92:81-90.

68. Holland PR, Akerma S, Goadsby PJ. Orexin 1 receptor activation attenautes neurogenic dural vasodialtion in an animal model of trigeminovascular nocicpetion. J Pharm Exp Ther. 2005;315:1380-5.

69. Holland PR, Akerman S, Goadsby PJ. Modulation of nociceptive dural input to the trigeminal nucleus caudalis via activation of the orexin 1 receptor in the rat. Eur J Neurosci. 2006;24:2825-33.

70. Benjamin L, Levy MJ, Lasalamdra MP, Knight YE, Akerman S, Classey JD, et al. Hypothalamic activation after stimulation of the superiro saggital sinus in the cat: a Fos study. Neurobiol Dis. 2004; 16:500-5.

71. Bartsch T, Levy MJ, Knight YE, Goadsby PJ. Differential modulation of nociceptive dural input to [hypocretin] orexin A and B receptor activation in the posterior hypothalamic area. Pain. 2004 Jun;109:367-78.

72. Gatfield J, Brisbare-Roch C, Jenck F, Boss C. Orexin receptor antagonists: A new concept in CNS disorders? ChemMedChem. 2010;5: $1197-214$.

73. Hoffman J, Supronsinchai W, Akerman S, Winrow CJ, Renger JJ, Hargreaves R, et al. Nociceptive trigeminal neurotransmission is inhibited by the dual orexin receptor antagonist DORA-12. Cephalalgia. 2013;33(8):8-9. Abstr OR14. IHC 2013, International Headache Congress, Boston, MA, USA, 6/27/13-6/30/13. 